\title{
First Incidence and Progression Study for Diabetic Retinopathy in Portugal, the RETINODIAB Study
}

\section{Evaluation of the Screening Program for Lisbon Region}

Marco Dutra Medeiros, MD, ${ }^{1,2,3}$ Edgar Mesquita, MSc, ${ }^{4}$ Luís Gardete-Correia, MD, ${ }^{1}$ Joaquim Moita, MD, Victor Genro, MD, ${ }^{1}$ Ana Luísa Papoila, MSc, PhD, ${ }^{5,6,7}$ Antónia Amaral-Turkman, PhD, ,8 João Filipe Raposo, $\mathrm{MD}, \mathrm{PhD}^{1,9}$

Purpose: To estimate the 5-year incidence and progression of diabetic retinopathy (DR) among persons with type 2 diabetes mellitus (DM).

Design: Population-based, prospective, cohort study.

Participants: The RETINODIAB (Study Group for Diabetic Retinopathy Screening) program was implemented in the Lisbon and Tagus Valley area between July 2009 and December 2014. A total of 109543 readable screening examinations were performed and corresponded to 56903 patients who attended the screening program at entry. A total of 30641 patients $(53.85 \%)$ had at least 1 further screening event within the study period and were included in the analysis.

Methods: Participants underwent two $45^{\circ}$ nonstereoscopic retinal digital photographs per eye according to RETINODIAB protocol. All images were graded according to the International Clinical Diabetic Retinopathy Scale. Referable diabetic retinopathy (RDR) was defined for all patients graded as moderate nonproliferative DR (NPDR), severe NPDR, or proliferative DR (PDR), with or without maculopathy or mild NPDR with maculopathy. Nonparametric estimates of the annual and cumulative incidences were obtained by Turnbull's estimator. Associations between the potential risk factors and the time to onset/progression of retinopathy were assessed through a parametric survival analysis for interval-censored data.

Main Outcome Measures: The authors estimated the onset and progression incidence rates of DR.

Results: Yearly incidence of any DR in patients without retinopathy at baseline was $4.60 \%$ (95\% confidence interval $[\mathrm{Cl}], 3.96-4.76)$ in the first year, decreasing to $3.87 \%(95 \% \mathrm{Cl}, 2.57-5.78)$ in the fifth year. In participants with mild NPDR at baseline, the progression rate to RDR in year 1 was $1.18 \%$ (95\% Cl, 0.96-1.33). Incidence of any $\mathrm{DR}$ and RDR and DR progression rate were associated with known duration of diabetes, age at diagnosis, and use of insulin treatment.

Conclusions: This longitudinal epidemiologic study provides the first Portuguese incidence DR data in a large-scale population-based cohort of type 2 diabetes after a 5-year follow-up. Duration of diabetes, age at diagnosis, and insulin treatment were associated with increasing risk of incidence and progression of DR. A personalized schedule distribution of screening intervals according to the individual patient's profile should be implemented, with resulting benefits in terms of health costs. Ophthalmology 2015;122:2473-2481 @ 2015 by the American Academy of Ophthalmology.

Diabetic retinopathy (DR) remains a major cause of visual impairment and represents an ongoing and worrying worldwide public health issue. ${ }^{1-3}$ In 2013,382 million people had diabetes; this number is expected to increase to 592 million by 2035 , according to the International Diabetes Federation. ${ }^{4}$ Most people with diabetes live in low- and middle-income countries, and these will experience the greatest increase in cases of diabetes over the next 22 years. ${ }^{5}$

Diabetic retinopathy is a highly specific vascular complication of both type 1 and 2 diabetes, with incidence strongly related to the duration of diabetes. ${ }^{6-8}$ It is well established that effectiveness of the laser treatment depends on the accurate and timely treatment of DR among patients with diabetes mellitus (DM), particularly those with a high risk of DR.

This type of retinopathy represents an excellent paradigm for screening as laid out in the principles for screening of human disease described by Wilson and Jungner in $1968 .{ }^{10}$ Indeed, from a public health standpoint, screening for DR has been shown to be cost-effective in health economic terms. ${ }^{11}$

To decrease by approximately $30 \%$ the new cases of blindness caused by diabetes, the declaration of St. Vincent 
(1989) called for the implementation of national strategies for DR screening in a systematic manner. ${ }^{12}$ The World Health Organization, the International Diabetes Federation, and the Portuguese Directorate-General of Health (DGS) co-organized in 1997 the Fourth Meeting in Lisbon for the Implementation of the St. Vincent Declaration, which was attended by delegates from 60 countries. ${ }^{13}$ This conference reinforced, once again, the need for greater engagement from all signatory countries. This international challenge was strengthened at the Liverpool meeting in $2005 .^{14}$ Despite all this, it has been only in the last decade that significant progress has been made in implementing DR screening programs.

Portugal currently has a population of 10.6 million, who are predominantly white, and the majority $(\sim 8.5$ million $)$ are located on the western coast. ${ }^{15}$ According to the National Observatory for Diabetes, approximately 1 million Portuguese have diabetes, the equivalent of $13 \%$ of the population aged 20 to 79 years (2013). ${ }^{16}$

After a pilot regional DR screening program that was launched in 2008, the Diabetic Retinopathy Screening Service for Lisbon and Tagus Valley, RETINODIAB, was commissioned and driven by the Portuguese Diabetes Association (APDP) in 2009. This screening program was supported by the Regional Health Administration of Lisbon and Tagus Valley under the auspices of the DGS, which is a public health branch of the Ministry of Health. The major aim of this project was to identify all undiagnosed sightthreatening DR to ensure timely onward referral to Lisbon area hospital eye services.

The APDP is the world's oldest diabetes association and a senior member of the International Diabetes Federation. Since its foundation in 1926, the APDP has been driven by a single overarching objective: to improve the quality of life of people with diabetes.

In Portugal, so far, there are no accurate data on the incidence of DR, based on a large-scale screening communitybased program, over a period of time.

We describe the first incidence and progression study for DR in a Portuguese population among persons with type 2 DM. We estimated the annual and cumulative DR incidence and the DR rate progression over a 5-year period, and explored the association between the development of retinopathy and its putative risk factors.

\section{Methods}

\section{The RETINODIAB Network}

RETINODIAB (Study Group for Diabetic Retinopathy Screening) is a telemedical screening system carried out by the APDP that has focused on clinical aspects of DR screening. Its primary aim was to promote the advance of knowledge on all aspects of DR through an active cooperation between ophthalmologists and other specialists, such as endocrinologists, internists, and neurologists. Currently, more than 200,000 people nationwide have benefited from integrated and specialized healthcare services provided by the APDP in a range of diabetes-related fields: diabetology, ophthalmology, pediatrics, nutrition, cardiology, podiatry, nephrology, urology, women's health, and mental health.
Working under the auspices of Regional Health Administration of Lisbon and the Tagus Valley protocol (Portuguese Ministry of Health), the Association's clinical services were integrated into the public healthcare network around Lisbon and Tagus Valley. Moreover, the APDP has fostered the development of important scientific studies in epidemiology and diabetology in Portugal. ${ }^{17-19}$ The APDP has carried out relevant scientific work in collaboration with international institutions of reference.

\section{Lisbon and Tagus Valley Area}

Lisbon and Tagus Valley is 1 of the 5 regions of Portugal termed as "Nomenclature of Territorial Units for Statistics" (NUTS II subdivisions). The region corresponds to $13 \%$ of the Portuguese territory including the capital, Lisbon; it has a population of 3.7 million ( $34 \%$ of the total population); and, according to the Centre for Regional Dynamics Observation (2009), it represents 44\% of the national gross domestic product.

There are 15 primary health center groups in this area, organized according to the 5 existing NUTS III (subregions: Greater Lisbon, Setubal Peninsula, Middle Tagus, Lezíria, and West Coast). Their mission is to ensure the provision of Primary Health Care in a particular geographic area, enhancing health gains accomplished by Family Health Units or other primary care units.

\section{Nonmydriatic Digital Camera for Diabetic Retinopathy Screening}

Currently, teleophthalmology based on digital imaging system provides a credible and efficient DR screening service. Indeed, it allows a sensitivity of $92 \%$ and a specificity of $90 \%$, thus justifying its use in terms of cost/benefit and gains to health. ${ }^{20}$ Furthermore, it offers several advantages, such as instant photography display, ease of storage and consultation of iconography available, and a remote transmission via Internet. The advance of telemedicine in ophthalmology screening of DR has a broader application, especially in remote ophthalmologic-assistance areas.

\section{Portuguese Diabetes Association Screening Protocol}

The RETINODIAB screening program was held in several health units covered by the APDP protocol. It encompasses several screening centers located in the primary care units of 15 primary health center groups. Each screening center is equipped with a nonmydriatic camera (model CR-2, Canon, Tokyo, Japan). Persons with type 2 diabetes who are registered with a general practitioner in the Lisbon and Tagus Valley area and not already under the care of hospital eye services for DR-related reasons are required to be referred to RETINODIAB program. Only patients with no history of documented DR or mild DR were referred for screening. All persons invited for examination are sent an appointment letter with a date, time, and medical center for screening. The devices were allocated-geographic distribution among different health centers-in a regular and systematic manner to improve the interaction with general practitioners, thus optimizing system referral and screening. All images were classified according to The International Clinical Diabetic Retinopathy Scale. ${ }^{21}$

This International Clinical Classification System categorizes DR severity in 5 levels, which include 3 stages of low risk (none, mild, and moderate NPDR), a fourth stage of severe NPDR, and a fifth stage of PDR, in the presence or absence of diabetic macular edema, which is graded separately. Referable diabetic retinopathy (RDR) was defined for all patients graded as moderate NPDR; severe NPDR or PDR, with or without maculopathy; or mild NPDR with maculopathy. This category (RDR) relates to those 
Dutra Medeiros et al · Diabetic Retinopathy in Portugal

Table 1. Baseline Characteristics of Study Participants

\begin{tabular}{|c|c|c|c|}
\hline Characteristics & 1 Screening Event $(n=26262)$ & $>1$ Screening Event $(\mathrm{n}=30641)$ & $P$ Value \\
\hline Age (yrs): median $\left(\mathrm{P}_{25} ; \mathrm{P}_{75}\right)$ & $70(62 ; 77)$ & $70(62 ; 77)$ & 0.111 \\
\hline Known duration of DM (yrs): median $\left(\mathrm{P}_{25} ; \mathrm{P}_{75}\right)$ & $6(3 ; 12)$ & $9(5 ; 14)$ & $<0.001$ \\
\hline Age at diagnosis of $\mathrm{DM}(\mathrm{yrs})$ : median $\left(\mathrm{P}_{25} ; \mathrm{P}_{75}\right)$ & $61(52 ; 69)$ & $61(54 ; 69)$ & $<0.001$ \\
\hline \multicolumn{4}{|l|}{$\operatorname{Sex}(n ; \%)$} \\
\hline Male & $12982(49.4)$ & $15588(50.9)$ & 0.686 \\
\hline Female & $13280(50.6)$ & $15053(49.1)$ & \\
\hline \multicolumn{4}{|l|}{ Insulin $(\mathrm{n} ; \%)$} \\
\hline No & $25095(95.6)$ & $29259(95.5)$ & $<0.001$ \\
\hline Yes & $1166(4.4)$ & $1382(4.5)$ & \\
\hline $\mathrm{DM}=$ diabetes mellitus. & & & \\
\hline
\end{tabular}

who would, according to the guidelines, need referral to the hospital eye service for further clinical evaluation.

Because clinically significant macular edema is not discernible on nonstereoscopic images, maculopathy was defined as the presence of hard exudates within 1 disc diameter of the fovea. Patients found to have undergone panretinal laser treatment were classified as having PDR. Both eyes were assessed for DR, and the worse grade from the 2 eyes was used in the analysis. Digital retinal images were considered not gradable if the retina of both eyes could not be visualized properly, that is, retinal vessels were not visible within 1 disc diameter of the fovea and fine vessels were not visible across the surface of the optic disc. When only 1 eye was gradable, the presence or absence of DR relied on this eye and subsequently documented in RETINODIAB data. The reader automatically generated report displays of diagnosis of DR level, diagnosis of nondiabetic ocular disorders, and recommendations for follow-up. After the capture of images, they are compressed in the Digital Imaging and Communications in Medicine protocol and transmitted through the Internet to the central medical server for interpretation and storage. In the datasheet adjunct to retinography, the following information was documented: sex, patient age, duration of diabetes, treatment adopted (by diet, oral hypoglycemic agents, or insulin), metabolic control analysis by the last value of hemoglobin A1c (when available), and associated comorbidity.

Individuals having no DR signs in either eye at baseline and having any DR grade in either of the eyes at 1 of the 5 years of follow-up were considered incident cases. Progression of DR occurred for those patients with mild NPDR without maculopathy and having progressed to moderate or severe NPDR grade, PDR grade, maculopathy stage, or laser photocoagulation treatment during follow-up time.

\section{Fundus Photography Acquisition Method}

All orthoptists involved in the project received a full day of theoretic teaching and 5 days of practical training that addressed the operation of the nonmydriatic camera and matching software.

The retinography was performed in a dark room (a scotopic environment) to obtain a sufficient mydriasis. Each screening examination lasted approximately 10 minutes. Two $45^{\circ}$ nonstereoscopic retinal digital photographs per eye were obtained, one centered on the posterior pole and the other centered on the optic disc. Good patient compliance and clear optical media were crucial for obtaining good-quality pictures. Despite all efforts, in several patients it was impossible to obtain an image with minimum quality to be evaluated by the ophthalmologist. In these specific cases, they proceeded to iatrogenic pupil dilation with a topical mydriatic. Otherwise, the remaining possible causes for deficient acquisition of fundus were documented by technicians and proceeded to onward referral for scheduling with a specialist within a maximum period of 3 months, according to the protocol.

\section{APDPSoft Software}

The APDPSoft is a software developed since 1999 that has accompanied the evolution of the services provided by the APDP. Currently, this software supports and monitors a number of valences, especially in terms of clinical data file, markings management, laboratory parameters, invoicing the health subsystems, integration of numerous pieces of diagnosis equipment, and an effective liaison with the electronic services of the Ministry of Health (including the receipt of screening requests by the general

Table 2. Baseline Characteristics of Patients According to Diabetic Retinopathy Grading

\begin{tabular}{|c|c|c|c|c|c|}
\hline Characteristics & $\begin{array}{l}\text { No Retinopathy } \\
(\mathrm{n}=48 \text { 888) }\end{array}$ & $\begin{array}{c}\text { Any Retinopathy } \\
(\mathrm{n}=8015)\end{array}$ & $\begin{array}{c}P \\
\text { Value }\end{array}$ & $\begin{array}{l}\text { Referable Retinopathy } \\
\quad(\mathrm{n}=2910)\end{array}$ & $P$ Value \\
\hline Age $(y r s)$ : median $\left(\mathrm{P}_{25} ; \mathrm{P}_{75}\right)$ & $70(62 ; 77)$ & $70(62 ; 77)$ & 0.748 & $70(63 ; 76)$ & $<0.001$ \\
\hline Known duration of DM (yrs): median $\left(\mathrm{P}_{25} ; \mathrm{P}_{75}\right)$ & $5(2 ; 10)$ & $12(8 ; 20)$ & $<0.001$ & $15(10 ; 20)$ & $<0.001$ \\
\hline Age at diagnosis of $\mathrm{DM}(\mathrm{yrs})$ : median $\left(\mathrm{P}_{25} ; \mathrm{P}_{75}\right)$ & $62(54 ; 70)$ & $55(47 ; 63)$ & $<0.001$ & $53(44 ; 60)$ & $<0.001$ \\
\hline \multicolumn{6}{|l|}{$\operatorname{Sex}(n ; \%)$} \\
\hline Male & $24298(49.7)$ & $4272(53.3)$ & $<0.001$ & $1562(53.7)$ & $<0.001$ \\
\hline Female & $24590(50.3)$ & $3743(46.7)$ & & $1348(46.3)$ & \\
\hline \multicolumn{6}{|l|}{ Insulin $(\mathrm{n} ; \%)$} \\
\hline No & $47667(97.5)$ & $6963(86.9)$ & $<0.001$ & $2397(82.4)$ & $<0.001$ \\
\hline Yes & $1220(2.5)$ & $1052(13.1)$ & & $513(17.5)$ & \\
\hline
\end{tabular}


Table 3. Yearly Incidence and 95\% Confidence Interval of Any and Referable Retinopathy in Participants without Retinopathy at Baseline

\begin{tabular}{|c|c|c|c|c|c|c|c|c|}
\hline \multirow[b]{2}{*}{$\begin{array}{l}\text { Time from Last } \\
\text { Negative Screen }\end{array}$} & \multicolumn{4}{|c|}{ Any Retinopathy } & \multicolumn{4}{|c|}{ Referable Retinopathy } \\
\hline & $\begin{array}{l}\text { No. Entering } \\
\text { Time Interval }\end{array}$ & $n$ & $\begin{array}{c}\text { Annual } \\
\text { Incidence* (\%) }\end{array}$ & $\begin{array}{c}\text { Cumulative } \\
\text { Incidence* (\%) }\end{array}$ & $\begin{array}{l}\text { No. Entering } \\
\text { Time Interval }\end{array}$ & $n$ & $\begin{array}{c}\text { Annual } \\
\text { Incidence* (\%) }\end{array}$ & $\begin{array}{c}\text { Cumulative } \\
\text { Incidence* (\%) }\end{array}$ \\
\hline $1 \mathrm{yr}$ & 409 & 101 & $4.60(3.96-4.76)$ & $4.60(4.35-4.85)$ & 339 & 1 & $0.23(0.13-0.28)$ & $0.23(0.17-0.28)$ \\
\hline 2 yrs & 1629 & 246 & $2.40(2.17-3.09)$ & $7.00(6.67-7.34)$ & 1495 & 31 & $0.48(0.36-0.63)$ & $0.71(0.59-0.83)$ \\
\hline $3 \mathrm{yrs}$ & 4516 & 643 & $1.70(1.10-2.70)$ & $8.71(8.29-9.11)$ & 4215 & 42 & $0.32(0.07-0.54)$ & $1.03(0.87-1.19)$ \\
\hline $4 \mathrm{yrs}$ & 6788 & 530 & $1.89(0.63-2.87)$ & $10.59(10.02-11.16)$ & 6766 & 67 & $0.34(0.09-0.63)$ & $1.37(1.13-1.60)$ \\
\hline $5 \mathrm{yrs}$ & 13745 & 444 & $3.87(2.57-5.78)$ & $14.47(13.01-15.90)$ & 14272 & 55 & $0.002(0.00-0.04)$ & $1.37(1.13-1.60)$ \\
\hline
\end{tabular}

The 95\% CIs for annual incidences were obtained using bootstrap and for cumulative incidences using the function "survfit" from the R package "survival." $\mathrm{n}=$ the number of positive cases in a given time-interval or the number of patients reaching end point.

*To estimate annual and cumulative incidences of onset or progression of DR, nonparametric Turnbull estimator, which takes into account intervalcensored data, had to be applied.

practitioner and the return of the retinography report, stock images, and new appointment proposal).

Furthermore, it stores clinical data and fundus photographs, and provides data to statistical processing. Safe access to the application is allowed by the use of a password. The stored images were downloaded by certified ophthalmologists at the RETINODIAB Reading Centre, comprising 3 readers.

\section{Statistical Methods}

Characteristics of study participants were described using the median and interquartile range (P25; P75) for continuous variables and percentages for categoric variables. The primary end points were "time to onset" and "time to progression" of DR. The presence/progression or absence/no progression of DR was determined after each screening event during the study period. Although it was supposed to occur annually, screening examination took place at different periods of time during the 5-year follow-up. For people who developed retinopathy (or progressed) between 2 screening events, the time to DR onset (or progression) is set among the 2 instants, and therefore the data are interval censored; for those who did not develop the disorder (or did not progress) by the final

Table 4. Yearly Incidence and 95\% Confidence Interval of Referable Retinopathy in Participants with Minimum Retinopathy at Baseline

\begin{tabular}{lcrcc}
\hline \multirow{2}{*}{$\begin{array}{c}\text { Time } \\
\text { from Last }\end{array} \begin{array}{c}\text { Regative } \\
\text { Progression }\end{array}$} & \begin{tabular}{c} 
No. Entering \\
\cline { 2 - 5 } Time Interval
\end{tabular} & \multicolumn{1}{c}{$n$} & $\begin{array}{c}\text { Annual } \\
\text { Incidence* }(\%)\end{array}$ & $\begin{array}{c}\text { Cumulative } \\
\text { Incidence* }\end{array}$ \\
\hline $1 \mathrm{yr}$ & 817 & 34 & $1.18(0.96-1.33)$ & $1.18(1.05-1.30)$ \\
$2 \mathrm{yrs}$ & 2025 & 94 & $0.50(0.26-0.76)$ & $1.68(1.52-1.85)$ \\
$3 \mathrm{yrs}$ & 6968 & 164 & $0.50(0.22-1.05)$ & $2.18(1.96-2.40)$ \\
$4 \mathrm{yrs}$ & 7384 & 162 & $1.89(1.18-2.42)$ & $4.07(3.62-4.52)$ \\
$5 \mathrm{yrs}$ & 13447 & 112 & $0.52(0.00-1.09)$ & $4.59(3.74-5.44)$
\end{tabular}

The 95\% CIs for annual incidences were obtained using bootstrap and for cumulative incidences using the function "survfit" from the R package "survival."

$\mathrm{n}=$ the number of positive cases in a given time-interval or the number of patients reaching end point.

*To estimate annual and cumulative incidences of onset or progression of DR, nonparametric Turnbull estimator, which takes into account intervalcensored data, had to be applied. screening event, the data are right censored. Accordingly, to estimate annual and cumulative incidences of onset/progression of DR, nonparametric Turnbull estimator for interval-censored data was applied. Bootstrap was used to obtain the $95 \%$ confidence intervals (CIs) for the annual incidences. We proceeded to a parametric survival analysis using Weibull proportional hazards regression model for interval-censored data. The aim of this analysis was to identify the variables that could influence the incidence of any retinopathy or RDR or further retinopathy progression to RDR. For both univariable and multivariable regression analyses, age at diagnosis and duration of diabetes were categorized into 4 groups: $40-49$ years, 50-59 years, 60-69 years, and $\geq 70$ years, and $<5$ years, $5-9$ years, $10-15$ years, and $>15$ years, respectively.

Crude and multivariable-adjusted hazard ratios were reported. A level of significance $\alpha=0.05$ was considered. All data were analyzed using STATA 13.0 (StataCorp. 2013. Stata Statistical Software: Release 13. StataCorp LP, College Station, TX) and R software (R: A Language and Environment for Statistical Computing, R Core Team, R Foundation for Statistical Computing, Vienna, Austria, year = 2014, http://www.R-project.org).

\section{Ethical Approval}

Approval was obtained from the APDP ethics committee.

\section{Results}

The RETINODIAB screening program was implemented by the APDP in the Lisbon and Tagus Valley area between July 2009 and December 2014. It included type 2 diabetic patients, diagnosed at more than 40 years of age. A total of 109543 readable screening examinations were performed, which corresponded to 56903 patients who attended the screening program at entry.

Overall, not all screening examinations resulted in assessable images. In this study, retinal photographs of at least 1 eye could not be graded in 2876 of the 59779 total screening patient examinations performed at entry (4.81\%). This subset of patients was not included in the final group used to calculate several incidence and progression rates.

There were 48888 patients ( $85.9 \%$ ) who had no DR at baseline and were at risk of incident DR; therefore, they were eligible for the 5-year follow-up incidence study. All patients who had 
Dutra Medeiros et al · Diabetic Retinopathy in Portugal

Table 5. Yearly Incidence and 95\% Confidence Interval of Any and Referable Retinopathy in Participants Using Insulin Treatment and without Retinopathy at Baseline

\begin{tabular}{|c|c|c|c|c|c|c|c|c|}
\hline \multirow[b]{2}{*}{$\begin{array}{l}\text { Time from Last } \\
\text { Negative Screen }\end{array}$} & \multicolumn{4}{|c|}{ Any Retinopathy } & \multicolumn{4}{|c|}{ Referable Retinopathy } \\
\hline & $\begin{array}{l}\text { No. Entering } \\
\text { Time Interval }\end{array}$ & $n$ & $\begin{array}{c}\text { Annual } \\
\text { Incidence* (\%) }\end{array}$ & $\begin{array}{c}\text { Cumulative } \\
\text { Incidence* (\%) }\end{array}$ & $\begin{array}{l}\text { No. Entering } \\
\text { Time Interval }\end{array}$ & $n$ & $\begin{array}{c}\text { Annual } \\
\text { Incidence* (\%) }\end{array}$ & $\begin{array}{c}\text { Cumulative } \\
\text { Incidence* (\%) }\end{array}$ \\
\hline $1 \mathrm{yr}$ & 23 & 10 & $12.47(10.43-14.81)$ & $12.47(10$ & 15 & 0 & $0.91(0.0$ & 0.91 \\
\hline $2 \mathrm{yrs}$ & 60 & 23 & $6.67(1.78-9.27)$ & $19.14(16.37-21.82)$ & 46 & 3 & $2.62(1.06-4.29)$ & $3.53(2.19-4.85)$ \\
\hline $3 \mathrm{yrs}$ & 140 & 57 & $4.83(0.33-9.12)$ & $23.97(20.56-27.23)$ & 108 & 5 & $0.002(0.00-1.40)$ & $3.53(2.20-4.85)$ \\
\hline 4 yrs & 284 & 46 & $0.00(0.00-6.05)$ & $23.97(20.56-27.24)$ & 283 & 13 & $1.93(0.00-4.34)$ & $5.46(3.20-7.67)$ \\
\hline $5 \mathrm{yrs}$ & 432 & 53 & $11.81(4.57-19.97)$ & $35.79(27.79-42.90)$ & 487 & 10 & $0.00(0.00-0.02)$ & $5.46(3.20-7.67)$ \\
\hline
\end{tabular}

The 95\% CIs for annual incidences were obtained using bootstrap and for cumulative incidences using the function "survfit" from the R package "survival." $\mathrm{n}=$ the number of positive cases in a given time-interval or the number of patients reaching end point.

*To estimate annual and cumulative incidences of onset or progression of DR, nonparametric Turnbull estimator, which takes into account intervalcensored data, had to be applied.

evidence of mild NPDR during the 5-year study period were eligible to estimate the DR rate progression.

A total of 26262 patients $(46.15 \%)$ were screened only once. Otherwise, 30641 patients $(53.85 \%)$ had at least 1 further screening event within the study period and were included in the analysis. In addition, we found that 14677 participants $(25.8 \%)$ had a third screening event, 6042 patients $(10.63 \%)$ had a fourth screening event, and 1151 patients $(2.03 \%)$ had a fifth screening event, corresponding to 83152 examinations performed. It was not possible to verify whether the nonattending patients had undergone ophthalmological examination elsewhere during the follow-up.

The baseline characteristics of study participants at risk of incidence or progression of DR are shown in Tables 1 and 2. Table 3 shows the annual and cumulative incidence for development of any retinopathy and RDR for patients without retinopathy at baseline. Yearly incidence of any DR in patients without retinopathy at baseline was $4.60 \%$ (95\% CI, 3.96-4.76) in the first year, decreasing to $3.87 \%$ (95\% CI, 2.57-5.78) in the fifth year; the cumulative incidence at 5 years was $14.47 \%(95 \%$ CI, 13.01-15.90).

Table 6. Yearly Incidence and 95\% Confidence Interval of Referable Retinopathy in Participants Using Insulin Treatment and with Minimum Retinopathy at Baseline

\begin{tabular}{lcrcc}
\hline \multirow{2}{*}{$\begin{array}{c}\text { Time } \\
\text { from Last } \\
\text { Negative }\end{array}$} & \multicolumn{4}{c}{ Referable Retinopathy } \\
\cline { 2 - 5 } $\begin{array}{c}\text { Progression } \\
\text { Pro. Entering }\end{array}$ & $\begin{array}{c}\text { Time Interval } \\
n\end{array}$ & $\begin{array}{c}\text { Annual } \\
\text { Incidence* }\end{array}$ & $\begin{array}{c}\text { Cumulative } \\
\text { Incidence* }\end{array}$ \\
\hline $1 \mathrm{yr}$ & 67 & 4 & $4.34(2.55-5.65)$ & $4.34(3.21-5.46)$ \\
$2 \mathrm{yrs}$ & 107 & 19 & $1.78(0.28-4.03)$ & $6.12(4.68-7.53)$ \\
$3 \mathrm{yrs}$ & 265 & 20 & $5.82(0.01-8.33)$ & $11.94(9.30-14.50)$ \\
$4 \mathrm{yrs}$ & 428 & 35 & $2.96(0.00-8.54)$ & $14.90(11.64-18.03)$ \\
$5 \mathrm{yrs}$ & 515 & 25 & $0.00(0.00-0.00)$ & $14.90(11.64-18.03)$
\end{tabular}

The 95\% CIs for annual incidences were obtained using bootstrap and for cumulative incidences using the function "survfit" from the R package "survival."

$\mathrm{n}=$ the number of positive cases in a given time-interval or the number of patients reaching end point.

*To estimate annual and cumulative incidences of onset or progression of DR, nonparametric Turnbull estimator, which takes into account intervalcensored data, had to be applied.
The annual incidence of RDR at 1 year was $0.23 \%$ (95\% CI, $0.13-0.28)$, decreasing to $0.002 \%$ (95\% CI, 0.00-0.04) in the fifth year, with a cumulative incidence of $1.37 \%$ (95\% CI, 1.13-1.60).

In participants with mild NPDR at baseline, the progression rate to RDR in the first year was $1.18 \%$ (95\% CI, 0.96-1.33) and $0.52 \%(95 \% \mathrm{CI}, 0.00-1.09)$ in the fifth year, with a cumulative incidence of $4.59 \%$ (95\% CI, 3.74-5.44) to higher grades of retinopathy after 5 years (Table 4$)$.

In the subgroup of patients under insulin treatment and without retinopathy at baseline, the yearly incidence of any retinopathy was $12.47 \%$ (95\% CI, 10.43-14.81) in the first year, decreasing to $11.81 \%$ (95\% CI, 4.57-19.97) in the fifth year (Table 5). The cumulative incidence at 5 years was $35.79 \%(95 \% \mathrm{CI}$, $27.79-42.90)$. The yearly progression DR rate was $4.34 \%(95 \%$ CI, 2.55-5.65) in the first year, decreasing to $2.96 \%(95 \% \mathrm{CI}$, 0.00-8.54) in the fourth year in patients receiving insulin treatment and with minimum retinopathy at entry (Table 6). Furthermore, the cumulative incidence of DR progression was $14.9 \%$ (95\% CI, 11.64-18.03) in participants who received insulin treatment.

Table 7 highlights the effect of known duration of DM, age at diagnosis of DM, and insulin treatment on the risk of participants developing any or referable retinopathy. A significantly increased risk of RDR was associated with an increased duration of DM. All factors were significant except for the subgroup of patients with a known duration of DM between 5 and 9 years for adjusted RDR. These results show that hazard rate increases over time; patients diagnosed at less than 50 years of age and with more than 15 years of diabetes duration are at a higher risk of developing the disease. An earlier age at diagnosis, a long duration of diabetes, and insulin treatment had similar effects on the risk of developing any or referable retinopathy, according to the different subgroups.

Table 8 refers to the survival analysis considering participants with mild retinopathy at baseline who developed RDR. All factors were statistically significant, concerning all subgroups analyzed. A significant risk of referral was associated with an increased duration of DM, with a lower age at diabetes diagnosis and in the subset of patients receiving insulin therapy in the 5 years analyzed. In this Weibull proportional hazards regression model, individuals who were diagnosed at baseline with mild NPDR at $<50$ years of age were significantly more likely to 
Table 7. Parametric Survival Analysis Considering Participants without Retinopathy at Baseline Who Developed Any and Referable Retinopathy

\begin{tabular}{|c|c|c|c|c|}
\hline \multirow[b]{2}{*}{ Putative Risk Factor } & \multicolumn{2}{|c|}{ Any Retinopathy } & \multicolumn{2}{|c|}{ Referable Retinopathy } \\
\hline & Crude HR (95\% CI) & Adjusted HR (95\% CI) & Crude HR (95\% CI) & Adjusted HR $(95 \% \mathrm{CI})$ \\
\hline \multicolumn{5}{|l|}{ Known duration of DM } \\
\hline$<5 \mathrm{yrs}$ & 1.00 & 1.00 & 1.00 & 1.00 \\
\hline $5-9$ yrs & $1.35(1.18-1.55)$ & $1.24(1.08-1.42)$ & $1.77(1.05-2.98)$ & $1.60(0.95-2.69)$ \\
\hline $10-15$ yrs & $1.77(1.55-2.03)$ & $1.48(1.29-1.70)$ & $2.95(1.79-4.87)$ & $2.33(1.41-3.86)$ \\
\hline$>15$ yrs & $2.81(2.44-3.25)$ & $2.07(1.79-2.41)$ & $5.91(3.57-9.78)$ & $3.87(2.31-6.48)$ \\
\hline \multicolumn{5}{|l|}{ Age at diagnosis of DM } \\
\hline$\geq 70$ yrs & 1.00 & 1.00 & 1.00 & 1.00 \\
\hline $60-69$ yrs & $1.47(1.24-1.74)$ & $1.41(1.19-1.66)$ & $2.39(1.23-4.62)$ & $2.19(1.13-4.24)$ \\
\hline $50-59$ yrs & $2.61(2.23-3.05)$ & $2.38(2.04-2.79)$ & $3.63(1.92-6.86)$ & $3.04(1.60-5.76)$ \\
\hline$<50$ yrs & $4.26(3.62-5.02)$ & $3.45(2.92-4.07)$ & $8.42(4.45-15.93)$ & $5.66(2.97-10.80)$ \\
\hline \multicolumn{5}{|l|}{ Insulin } \\
\hline No & 1.00 & 1.00 & 1.00 & 1.00 \\
\hline Yes & $2.90(2.50-3.37)$ & $2.17(2.92-4.07)$ & $4.49(3.06-6.60)$ & $3.04(2.06-4.49)$ \\
\hline
\end{tabular}

$\mathrm{CI}=$ confidence interval; $\mathrm{DM}=$ diabetes mellitus; $\mathrm{HR}=$ hazard ratio.

The following parameters were used for adjustment: duration of DM, age at diagnosis of DM, and insulin treatment. All factors were statistically significant except "known duration of DM" between 5 and 9 years for referable retinopathy.

develop DR compared with individuals who were diagnosed at $\geq 70$ years of age (adjusted hazard ratio, $4.63 ; 95 \%$ CI, 3.17-6.77).

\section{Discussion}

Diabetes has a high prevalence in Portugal. The PREVADIAB study, ${ }^{18}$ which was carried out by the APDP, found a diabetes prevalence of $11.7 \%$, with a significant difference between men (14.2\%) and women $(9.5 \%)$. Although $6.6 \%$ of the subjects had previously been diagnosed with diabetes, $5.1 \%$ were undiagnosed. In addition, the APDP undertook another epidemiologic study to ascertain DR prevalence in a Portuguese

Table 8. Parametric Survival Analysis Considering Participants with Minimum Retinopathy at Baseline Who Developed Referable Retinopathy

\begin{tabular}{|c|c|c|}
\hline \multirow[b]{2}{*}{ Putative Risk Factor } & \multicolumn{2}{|c|}{ Referable Retinopathy } \\
\hline & Crude HR (95\% CI) & Adjusted HR $(95 \% \mathrm{CI})$ \\
\hline \multicolumn{3}{|l|}{ Known duration of DM } \\
\hline$<5$ yrs & 1.00 & 1.00 \\
\hline $5-9$ yrs & $1.73(1.26-2.39)$ & $1.52(1.10-2.10)$ \\
\hline $10-15$ yrs & $3.15(2.32-4.28)$ & $2.41(1.77-3.28)$ \\
\hline$>15$ yrs & $5.83(4.29-7.93)$ & $3.64(2.65-4.99)$ \\
\hline \multicolumn{3}{|c|}{ Age at diagnosis of DM } \\
\hline$\geq 70$ yrs & 1.00 & 1.00 \\
\hline $60-69$ yrs & $1.85(1.24-2.77)$ & $1.69(1.13-2.53)$ \\
\hline $50-59$ yrs & $4.06(2.80-5.88)$ & $3.28(2.26-4.76)$ \\
\hline$<50$ yrs & $7.35(5.06-10.67)$ & $4.63(3.17-6.77)$ \\
\hline \multicolumn{3}{|l|}{ Insulin } \\
\hline No & 1.00 & 1.00 \\
\hline Yes & $4.34(3.51-5.38)$ & $2.82(2.27-3.51)$ \\
\hline
\end{tabular}

$\mathrm{CI}=$ confidence interval; $\mathrm{DM}=$ diabetes mellitus; $\mathrm{HR}=$ hazard ratio. The following parameters were used for adjustment: duration of DM, age at diagnosis of DM, and insulin treatment. All factors were statistically significant. population-based cohort. In this study, we reported a prevalence of any DR, NRDR, and RDR in type 2 diabetic patients of $16.3 \%, 10.4 \%$, and $5.9 \%$, respectively. ${ }^{17}$

The teleophthalmology network constitutes an efficient means to overcome the lack of ophthalmologists. ${ }^{19}$ In accordance with these statistics, Portugal may have approximately 1 million people with diabetes, of whom 700000 are diagnosed and receiving medical treatment and who should be consulted annually according to the criteria stated earlier. According to the Portuguese Ophthalmology Society, each of the 930 Portuguese ophthalmologists might observe approximately 753 cases per year, an infeasible number in terms of logistics specialty requirements. Screening centers or mobile units using nonmydriatic cameras should be allocated in areas with a high rate of poverty and a low number of ophthalmologists, such as the West Region, which is covered by the RETINODIAB program (Fig 1). Furthermore, the centralization of the network around a central reading headquarters led to uniformity regarding the classification approach between graders. However, because of a standard multidisciplinary approach, general practitioners and endocrinologists could adjust their therapeutics without delay, when required. Since the publication of the DGS recommendations for DR screening, we have adopted a protocol that includes 2 nonmydriatic photographs centered on the macula and the disc. ${ }^{22}$

The epidemiologic cohort studies addressing DR incidence have varied worldwide, at least partly because of different ethnic populations. Although most observational studies have adopted annual screening protocols, the screening intervals and strategies for grading retinopathy have differed, yielding to an inaccurate comparison of the DR incidence rates among published data.

The long-term incidence of DR was addressed in several western populations, such as in the Wisconsin Epidemiologic Study of Diabetic Retinopathy, ${ }^{23}$ the Fyn County Study, ${ }^{24}$ and an English population-based cohort. ${ }^{25}$ 


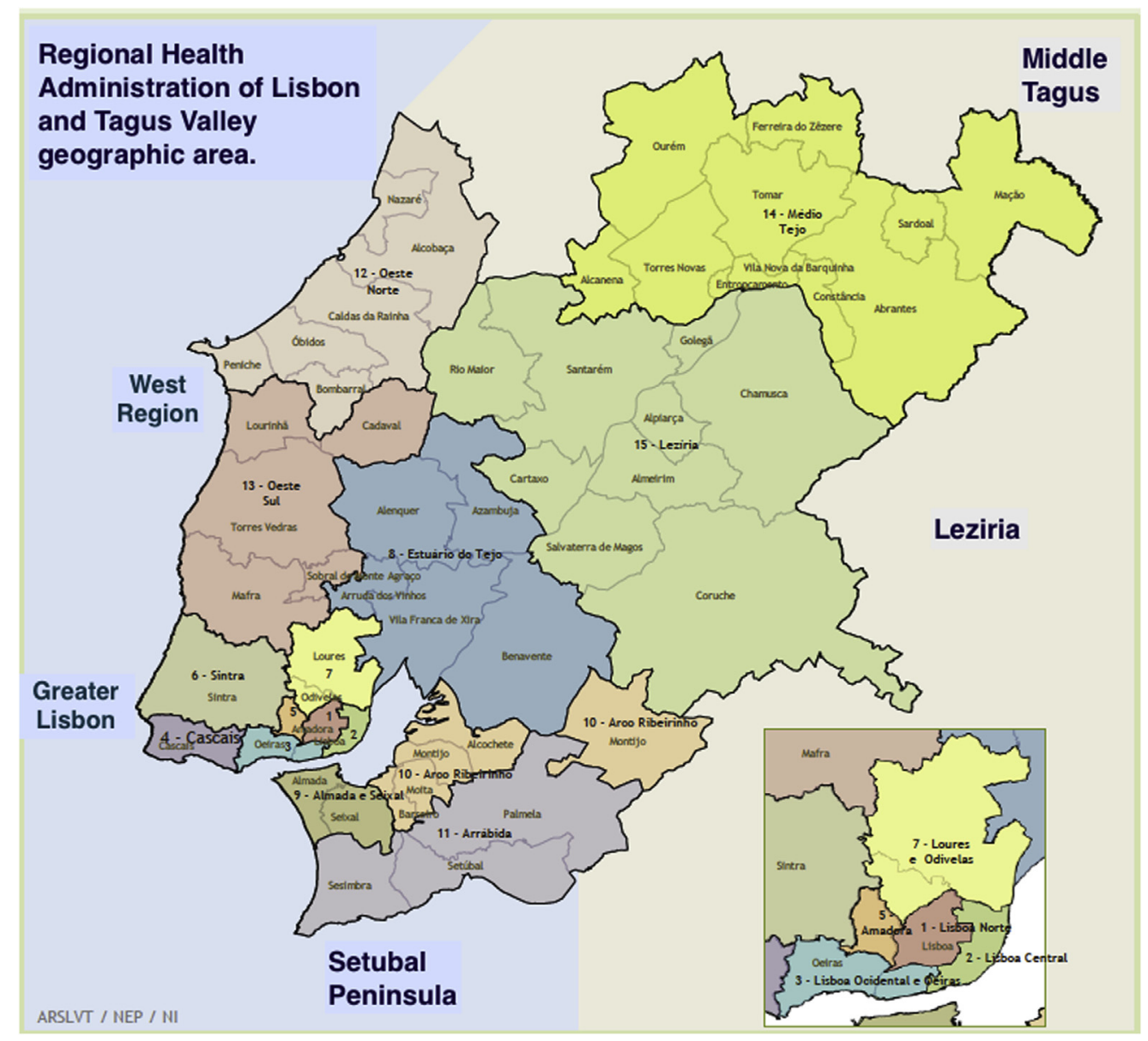

Figure 1. Regional Health Administration of the Lisbon and Tagus Valley geographic area.

In the Wisconsin Epidemiologic Study of Diabetic Retinopathy, Klein et $\mathrm{al}^{23}$ analyzed the progression of retinopathy in persons with type 1 diabetes after 25 years of follow-up. They ascertained a 25-year cumulative rate of DR progression of $83 \%$, a progression to PDR of $42 \%$, and an improvement of DR of $18 \%$. In addition, the 25 -year cumulative incidence was $29 \%$ for macular edema and $17 \%$ for clinically significant macular edema.

In England, 20686 patients with type 2 diabetes without PDR or sight-threatening maculopathy at baseline have been followed during a 17 -year period. ${ }^{25}$ After 5 years, few patients without retinopathy at baseline developed preproliferative retinopathy (cumulative incidence 4.0\%), sight-threatening maculopathy $(0.59 \%)$, or PDR $(0.68 \%)$. After 10 years of follow-up, the respective cumulative incidences were $16.4 \%, 1.2 \%$, and $1.5 \%$. Among those with NPDR (background) at baseline, after 5 years $23 \%$ developed pre-proliferative retinopathy, $5.2 \%$ developed maculopathy, and $6.1 \%$ developed PDR; after 10 years, the respective cumulative incidences were $53 \%, 9.6 \%$, and $11 \%$.

The Liverpool Diabetic Eye Study screening program reported an annual incidence of RDR of $0.2 \%$ in the first year, with a cumulative incidence of $1.7 \%$ at 4 years. ${ }^{26}$ The authors recommended an extension of the screening interval to triennial screening, based on the $95 \%$ probability of people remaining free from sight-threatening retinopathy with a mean screening interval of 5.4 years.

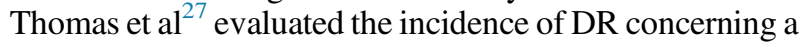
Welsh national-based diabetic-cohort screening that included 57199 people with type 2 diabetes who had no evidence of DR at baseline, over a 4-year period. They reported a cumulative incidence of any and referable retinopathy at 4 years of $360^{27}$ and 11.64 per 1000 people, respectively. In a Swedish screening program of a cohort with well-controlled type $2 \mathrm{DM}$ (patients without DR and with mean hemoglobin A1c $6.4 \%$ at baseline), the researchers observed that $28 \%$ of participants developed mild to moderate retinopathy but did not develop referable retinopathy in the form of severe preproliferative or PDR over a 3-year period. ${ }^{28}$

In the RETINODIAB cohort, the incidence of any DR in patients without retinopathy at baseline was $4.60 \%$ (95\% CI, $3.96-4.76$ ) in the first year, decreasing to $3.87 \%$ (95\% CI, 2.57-5.78) in the fifth year; the cumulative incidence at 5 years was $14.47 \%$ (95\% CI, 13.01-15.90). The annual incidence of referable retinopathy at 1 year was $0.23 \%(95 \%$ CI, $0.13-0.28$ ), increasing to $0.34 \%$ (95\% CI, 0.09-0.063) at 4 years, with a cumulative incidence of $1.37 \%(95 \% \mathrm{CI}$, $1.13-1.60)$. 
In patients with mild NPDR at baseline, the progression rate to RDR in the first year was $1.18 \%(95 \% \mathrm{CI}$, $0.96-1.33$ ) and $0.52 \%$ (95\% CI, 0.00-1.09) in the fifth year, with a cumulative incidence of $4.59 \%(95 \% \mathrm{CI}$, 3.74-5.44) to higher grades of retinopathy after 5 years.

The observed yearly and cumulative incident rates of RETINODIAB cohort were close to those in the previous published reports, namely, from the Liverpool Diabetic Eye Study screening program. Furthermore, our study reported an increasing hazard rate over time, with regard to a longer duration of diabetes, an earlier age at diagnosis, and under insulin treatment.

This was reinforced by other studies documenting that the incidence of DR was associated with a longer duration of diabetes and a younger age at diagnosis of diabetes. Although the duration of diabetes is not a modifiable risk characteristic, our findings might contribute to better management strategies by suggesting that patients with the features described have a higher risk of DR emergence and thus need more regular screening. This is in accordance with several studies that advise a customized schedule distribution of screening intervals according to the individual patient's risk. ${ }^{11,29-32}$ In fact, increasing the length of the screening intervals for lower-risk cases would involve fewer screening episodes, with obvious benefits in terms of health costs.

The data from the screening program in Iceland concluded that biennial screening would be safe in those people without evidence of DR at first screening examination. ${ }^{31}$ Therefore, on the basis of the current published literature, we should note that annual eye examination is not necessary for all patients with type 2 diabetes.

The main purpose of the RETINODIAB implementation was to improve DR screening in Lisbon and the Tagus Valley surrounding area to efficiently perform, within an acceptable time frame, all eye examinations according to the guidelines of the DGS. ${ }^{21}$ In addition to its major objective to provide access to an annual fundus examination to more diabetic patients, another goal of RETINODIAB network is to improve the quality of the reports produced.

This longitudinal epidemiologic study provides the first Portuguese incidence DR data in a large-scale populationbased cohort with type 2 diabetes that includes a mediumterm follow-up and several well-controlled risk factors. To the best of our knowledge, this study represents one of the largest reported community-based DR screening program to ascertain the incidence and progression of this disease. We will continue to follow these patients to better define all epidemiologic variables regarding this diabetic population.

Acknowledgments. The authors thank all the people involved in the RETINODIAB network: the graders, orthoptists, computer engineers, and administrative personnel.

\section{References}

1. Mokdad AH, Ford ES, Bowman BA, et al. Diabetes trends in the U.S.: 1990-98. Diabetes Care 2000;23:1278-83.
2. Guariguata L, Whiting DR, Hambleton I, et al. Global estimates of diabetes incidence for 2013 and projections for 2035. Diabetes Res Clin Pract 2014;103:137-49.

3. Williams R, Airey M, Baxter H, et al. Epidemiology of diabetic retinopathy and macular oedema: a systematic review. Eye (Lond) 2004;18:963-83. Review.

4. International Diabetes Federation. IDF Diabetes Atlas. 6th ed. Brussels, Belgium: International Diabetes Federation; 2013. Available at: http://www.idf.org/diabetesatlas. Accessed December 11, 2014.

5. Yang W, Lu J, Weng J, et al. China National Diabetes and Metabolic Disorders Study Group. Prevalence of diabetes among men and women in China. N Engl J Med 2010;362: 1090-101.

6. American Diabetes Association Standards of Medical Care in Diabetes, 2014. Diabetes Care 2014;37:S14-80.

7. Klein R. Hyperglycemia and microvascular and macrovascular disease in diabetes. Diabetes Care 1995;18:258-68.

8. Estacio RO, McFarling E, Biggerstaff $\mathrm{S}$, et al. Overt albuminuria predicts diabetic retinopathy in Hispanics with NIDDM. Am J Kidney Dis 1998;31:947-53.

9. Diabetic Retinopathy Study Research Group. Photocoagulation treatment of proliferative diabetic retinopathy: clinical application of Diabetic Retinopathy Study (DRS) findings report number 8. Ophthalmology 1981;88: 583-600.

10. Wilson JMG, Jungner G. Principles and Practice of Screening for Disease. WHO Chronicle 22. Geneva: World Health Organization; 1968:473. Public Health Papers, \#34.

11. Jones S, Edwards RT. Diabetic retinopathy screening: a systematic review of the economic evidence. Diabet Med 2010;27:249-56.

12. World Health Organization/International Foundation Europe. Diabetes care and research in Europe: the Saint Vincent declaration. Diabet Med 1990;7:360.

13. Fourth Meeting for the Implementation of the St. Vincent Declaration. Diabetes Care and Research in Europe. Improvement of Diabetes Care. Lisbon, Portugal, February 26 to March 1, 1997.

14. Screening for diabetic retinopathy in Europe: 15 years after the Saint Vincent Declaration. Proceedings of the Liverpool Declaration, November 17-18, 2005, Liverpool, UK. Available at: http://www.drscreening2005.org.uk. Accessed November 21, 2014.

15. Census - Final results: Portugal - 2011. Statistics Portugal. November 20, 2012. Available at: http://www.ine.pt. Accessed November 21, 2012.

16. Diabetes: Factos e Números 2013- Relatório Anual do Observatório Nacional da Diabetes; Novembro/2013. Available at: https://www.dgs.pt/documentos-e-publicacoes/diabetes-factos-e-numeros-2013.aspx. Accessed December 6, 2014.

17. Dutra Medeiros M, Mesquita E, Papoila AL, et al. First diabetic retinopathy prevalence study in Portugal: RETINODIAB Study-Evaluation of the screening programme for Lisbon and Tagus Valley region. Br J Ophthalmol 2015 Apr 2. pii: bjophthalmol-2015-306727. doi: 10.1136/bjophthalmol-2015306727. [Epub ahead of print].

18. Gardete-Correia L, Boavida JM, Raposo JF, et al. First diabetes incidence study in Portugal: PREVADIAB study. Diabet Med 2010;27:879-81.

19. Pinto-Figueiredo L, Moita J, Genro V, et al. Diabetic retinopathy in a population of 1,302 insulin dependent diabetics (IDDM) diagnosed before 30 years of age. Int Ophthalmol 1992;16:429-37. 
20. Massin P, Erginay A, Ben Mehidi A, et al. Evaluation of a new non-mydriatic digital camera for detection of diabetic retinopathy. Diabet Med 2003;20:635-41.

21. Wilkinson CP, Ferris FL 3rd, Klein RE, et al. Global Diabetic Retinopathy Project Group. Proposed international clinical diabetic retinopathy and diabetic macular edema disease severity scales. Ophthalmology 2003;110:1677-82.

22. Norma da DGS n ${ }^{\circ}$ 006/2011 de 27/01/2011. Diagnóstico Sistemático e Tratamento da Retinopatia Diabética. Available at: http://www.dgs.pt/ms/7/default.aspx $? \mathrm{pl}=\& \mathrm{id}=5519$ \&acess $=0$. Accessed December 4, 2014.

23. Klein R, Knudtson MD, Lee KE, et al. The Wisconsin Epidemiologic Study of Diabetic Retinopathy: XXII the twenty-five-year progression of retinopathy in persons with type 1 diabetes. Ophthalmology 2008;115:1859-68.

24. Grauslund J, Green A, Sjølie AK. Prevalence and 25 year incidence of proliferative retinopathy among Danish type 1 diabetic patients. Diabetologia 2009;52:1829-35.

25. Jones CD, Greenwood RH, Misra A, Bachmann MO. Incidence and progression of diabetic retinopathy during 17 years of a population-based screening program in England. Diabetes Care 2012;35:592-6.

26. Younis N, Broadbent DM, Vora JP, Harding SP. Incidence of sight-threatening retinopathy in patients with type 2 diabetes in the Liverpool Diabetic Eye study: a cohort study. Lancet 2003;361:195-200.

27. Thomas RL, Dunstan F, Luzio SD, et al. Incidence of diabetic retinopathy in people with type 2 diabetes mellitus attending the Diabetic Retinopathy Screening Service for Wales: retrospective analysis. BMJ 2012;344:e874.

28. Agardh E, Tababat-Khani P. Adopting 3-year screening intervals for sight-threatening retinal vascular lesions in type 2 diabetic subjects without retinopathy. Diabetes Care 2011;34:1318-9.

29. Taylor-Phillips S, Mistry H, Leslie R, et al. Extending the diabetic retinopathy screening interval beyond 1 year: systematic review. Br J Ophthalmol 2015 Jan 13. pii: bjophthalmol-2014305938. doi: 10.1136/bjophthalmol-2014-305938. [Epub ahead of print].

30. Echouffo-Tcheugui JB, Ali MK, Roglic G, et al. Screening intervals for diabetic retinopathy and incidence of visual loss: a systematic review. Diabet Med 2013;30:1272-92.

31. Olafsdottir E, Stefansson E. Biennial eye screening in diabetic patients without retinopathy. 10 year experience. Br J Ophthalmol 2007;91:1599-601.

32. Aspelund T, Thornórisdóttir O, Olafsdottir E, et al. Individual risk assessment and information technology to optimise screening frequency for diabetic retinopathy. Diabetologia 2011;54:2525-32.

\section{Footnotes and Financial Disclosures}

Originally received: June 8, 2015.

Final revision: August 3, 2015.

Accepted: August 4, 2015.

Available online: September 14, 2015.

Manuscript no. 2015-939.

${ }^{1}$ Portuguese Diabetes Association, Lisbon, Portugal.

${ }^{2}$ Department of Ophthalmology, Central Lisbon Hospital Center, Lisbon, Portugal.

${ }^{3}$ NOVA Medical School, Universidade NOVA de Lisboa, Lisbon, Portugal.

${ }^{4}$ University of Minho, Braga, Portugal.

${ }^{5}$ Statistics and Informatics Department, NOVA Medical School, Universidade NOVA de Lisboa, Lisbon, Portugal.

${ }^{6}$ Epidemiology and Statistics Unit, Research Centre, Central Lisbon Hospital Center, Lisbon, Portugal.

${ }^{7}$ CEAUL (Center of Statistics and Applications); University of Lisbon, Lisbon, Portugal.

${ }^{8}$ Department of Statistics and Operational Research, Faculty of Sciences, University of Lisbon, Lisbon, Portugal.

${ }^{9}$ Department of Public Health/CEDOC (Chronic Diseases Research Center), NOVA Medical School, Universidade NOVA de Lisboa, Lisbon, Portugal.

Financial Disclosure(s):

The author(s) have no proprietary or commercial interest in any materials discussed in this article.
The APDP (Portuguese Diabetes Association) screening network was supported by a grant from the Portuguese Ministry of Health.

A.L.P. and A.A.-T.: Sponsored by national funds through the Fundação Nacional para a Ciência e Tecnologia, Portugal - FCT (Fundação para a Ciência e Tecnologia) under the project PEst-OE/MAT/UI0006/2014.

Author Contributions:

Conception and design: Medeiros, Mesquita, Gardete-Correia, Moita, Genro, Papoila, Raposo

Data collection: Medeiros, Mesquita, Moita, Genro, Papoila, AmaralTurkman, Raposo

Analysis and interpretation: Medeiros, Mesquita, Genro, Papoila, AmaralTurkman, Raposo

Obtained funding: Not applicable

Overall responsibility: Medeiros, Gardete-Correia, Moita, Genro, Papoila, Raposo

Abbreviations and Acronyms:

APDP $=$ Portuguese Diabetes Association; $\mathbf{C I}=$ confidence interval; DGS = Portuguese Directorate-General of Health; $\mathbf{D M}=$ diabetes mellitus; $\mathbf{D R}=$ diabetic retinopathy; $\mathbf{N P D R}=$ nonproliferative diabetic retinopathy; $\mathbf{P D R}=$ proliferative diabetic retinopathy; $\mathbf{R D R}=$ referable diabetic retinopathy.

Correspondence:

Marco Dutra Medeiros, MD, Portuguese Diabetes Association, Rua Salitre 118, 1250-203, Lisbon, Portugal. E-mail: marcodutramedeiros@ gmail.com. 